\title{
DIFFSTOCHASTIC EQUIVALENT LINEARIZATION BASED ON THE FOKKER-PLANK EQUATION APPROACH
}

\author{
NguYen Dong AnH - NGUYen Duc Tinh \\ Institute of Mechanics, Hanoi Vietnam
}

\section{Introduction}

Stochastic equivalent linearization is the most popular approach to the approximate analysis of non-linear systems under random excitations. Over many years the original version of Gaussian equivalent linearization (GEL) has been developed by many authors, see e.g. [Atalik \& Utku, 1976], [Casciati \& Faraveilli, 1986], [Roberts \& Spanos, 1990], [Zhang et al., 1991], [Anh \& Schiehlen, 1995]. In order to improve the accuracy of GEL different techniques have been proposed, see e.g. $[6,7,8]$.

In the paper a technique for determining the coefficients of the linearized equivalent equation based on the Fokker-Plank equation approach is presented. The investigation is then applied to Duffing and Vanderpol oscillations under a zero mean Gaussian white noise.

\section{Equivalent linearization criterion}

Consider a single-degree-of-freedom mechanical system, whose motion is described by the equation:

$$
\ddot{x}+2 h \dot{x}+\omega^{2} x+\varepsilon f(x, \dot{x})=\sigma \dot{\xi}(t),
$$

wherein the symbols have their customary meanings, $f$ is a non-linear function of $x$ and $\dot{x}, \omega, h$, $\sigma$ are positive constants, and $\varepsilon$ is a positive parameter. The random excitation $\xi(t)$ is a Gaussian white noise process of unit intensity

$$
E(\dot{\xi}(t), \dot{\xi}(t+\tau))=\delta(\tau)
$$

where $E($.$) denotes the expectation operation, and \delta(\tau)$ is Dirac-Delta function.

Following the linearization method, we introduce new linear terms in the expression of the equation (2.1)

$$
\ddot{x}+(2 h+\varepsilon \mu) \dot{x}+\left(\omega^{2}+\varepsilon \lambda\right) x+\varepsilon(f(x, \dot{x})-\mu \dot{x}-\lambda x)=\sigma \dot{\xi}(t)
$$

The linearized equation takes the form

$$
\ddot{x}+(2 h+\varepsilon \mu) \dot{x}+\left(\omega^{2}+\varepsilon \lambda\right) x=\sigma \dot{\xi}(t)
$$

There are some criteria for determining the coefficients $\mu, \lambda$, see e.g. $[6,7,8]$. In the paper an alternative approach to GEL is proposed as follows. 
According to the classical approach of the averaging method the state coordinates $(x, \dot{x})$ are transformed to the pair of amplitude and phase $(a, \varphi)$ by the change

$$
x(t)=a \cos \varphi, \quad \dot{x}(t)=-a \omega \sin \varphi .
$$

The Ito differential equations for $a$ and $\varphi$ are obtained from (2.3) [Mitropolskii et all., 1992]

$$
\begin{aligned}
d a= & \left\{\frac{1}{\omega}[(2 h+\varepsilon \mu) \dot{x}+\varepsilon \lambda x] \sin \varphi\right. \\
& \left.+\frac{\varepsilon}{\omega}(f-\lambda x-\mu \dot{x}) \sin \varphi+\frac{\sigma^{2}}{2 \omega^{2} a} \cos ^{2} \varphi\right\} d t-\frac{\sigma}{\omega} \sin \varphi d \xi(t), \\
d \varphi= & \left\{\omega+\frac{1}{a \omega}[((2 h+\varepsilon \mu) \dot{x}+\varepsilon \lambda x) \cos \varphi\right. \\
& \left.+\varepsilon(f-\lambda x-\mu \dot{x}) \cos \varphi]-\frac{\sigma^{2}}{2 \omega^{2} a^{2}} \sin ^{2} \varphi\right\} d t-\frac{\sigma}{\omega a} \cos \varphi d \xi(t)
\end{aligned}
$$

The Fokker - Plank equation (FP equation) for the stationary probability density function $W(a, \varphi)$ corresponding to the system $(2.6)$ takes the form:

$$
\begin{aligned}
A_{n}[W]= & \omega \frac{\partial W}{\partial \varphi}+\left[K_{i}, K_{i f}\right] L(W)+\varepsilon\left\{\frac{\partial}{\partial a}\left[\frac{1}{\omega}(f-\lambda x-\mu \dot{x}) \sin \varphi W\right]\right. \\
& \left.+\frac{\partial}{\partial \varphi}\left[\frac{1}{a \omega}(f-\lambda x-\mu \dot{x}) \cos \varphi W\right]\right\}=0,
\end{aligned}
$$

where $\left[K_{i}, K_{i j}\right] L$ denotes the following linear differential operator

$$
\begin{aligned}
{\left[K_{i}, K_{i j}\right] L[W]=} & \frac{\partial}{\partial a}\left(K_{1} W\right)+\frac{\partial}{\partial \varphi}\left(K_{2} W\right) \\
& -\frac{1}{2}\left[\frac{\partial^{2}}{\partial a^{2}}\left(K_{11} W\right)+2 \frac{\partial^{2}}{\partial a \partial \varphi}\left(K_{12} W\right)+\frac{\partial^{2}}{\partial \varphi^{2}}\left(K_{22} W\right)\right]
\end{aligned}
$$

and

$$
\begin{aligned}
K_{1} & =\frac{\sigma^{2}}{4 \omega^{2} a}-\left(h+\frac{\varepsilon \mu}{2}\right) a+\left[\frac{\sigma^{2}}{4 \omega^{2} a}+\left(h+\frac{\varepsilon \mu}{2}\right) a\right] \cos 2 \varphi+\frac{\varepsilon \lambda}{2 \omega} a \sin 2 \varphi, \\
K_{2} & =\frac{\varepsilon \lambda}{2 \omega}+\frac{\varepsilon \lambda}{2 \omega} \cos 2 \varphi-\left(h+\frac{\varepsilon \mu}{2}+\frac{\sigma^{2}}{2 \omega^{2} a^{2}}\right) \sin 2 \varphi, \\
K_{11} & =\frac{\sigma^{2}}{2 \omega^{2}}(1-\cos 2 \varphi) ; \quad K_{12}=\frac{\sigma^{2}}{2 \omega^{2} a} \sin 2 \varphi ; \quad K_{22}=\frac{\sigma^{2}}{2 \omega^{2} a^{2}}(1+\cos 2 \varphi) .
\end{aligned}
$$

Let $W_{1}(a, \lambda, \mu)$ be a solution of the FP equation corresponding to the linearized systems (2.4). If $A_{n}\left[W_{L}(a, \lambda, \mu)\right]=0$, then $W_{L}(a, \lambda, \mu)$ is also a solution of the FP equation (2.7) which corresponds to the non-linear systems (2.3). However, it is seen from (2.7) that in general $A_{n}\left[W_{L}(a, \lambda, \mu)\right]$ differs from zero. So, we minimise the error in mean square as follows

$$
\int_{0}^{2 \pi} \int_{0}^{2 \pi}\left\{A_{n}\left[W_{L}(a, \lambda, \mu)\right]\right\}^{2} d a d \varphi \rightarrow \min _{\lambda, \mu}
$$

The FP equation corresponding to the linearized equation (2.4) gives the solution

$$
W_{L}(a, \lambda, \mu)=c a \exp \left\{-\frac{\left(\omega^{2}+\varepsilon \lambda\right)(2 h+\varepsilon \mu)}{\sigma^{2}} a^{2}\right\},
$$


where $c$ is a normalization constant.

Substituting $(2.11)$ into $(2.10)$ one gets

$$
\begin{aligned}
H(\lambda, \mu)= & \int_{0}^{2 \pi} \int_{0}^{2 \pi}\left\{\frac{\partial}{\partial t}\left[\frac{1}{\omega}(f-\lambda x-\mu \dot{x}) \sin \varphi W_{1}\right]\right. \\
& \left.+\frac{\partial}{\partial \varphi}\left[\frac{1}{a \omega}(f-\lambda x-\mu \dot{x}) \cos \varphi W_{1}\right]\right\}^{2} d a d \varphi \rightarrow \min _{\lambda, \mu}
\end{aligned}
$$

The condition (2.12) gives a criterion for determining the linearization coefficients $\lambda$ and $\mu$.

\section{Application}

In order to elucidate this approach we consider two oscillators with non-linear stiffness and damping, under white noise excitation.

\subsection{Dufing oscillator}

Consider the following equation

$$
\ddot{x}+2 h \dot{x}+\omega^{2} x+\varepsilon \gamma x^{3}=\sigma \dot{\xi}(t)
$$

where $\gamma=$ const. In this case one gets

$$
f(x, \dot{x})=\gamma x^{3}
$$

and the linearized equation is

$$
\ddot{x}+2 h \dot{x}+\left(\omega^{2}+\varepsilon \lambda\right) \dot{x}=\sigma \dot{\xi}(t)
$$

So, ones gets probability density function (2.11) in the form

$$
W_{L}(a, \lambda)=c a \exp \left\{-\frac{\left(\omega^{2}+\varepsilon \lambda\right) 2 h}{\sigma^{2}} a^{2}\right\}
$$

Substituting (3.2), (3.4) into (2.12) and using (2.5), after some calculations one gets:

$$
H(a)=\pi c^{2}\left(\frac{5 \gamma^{2} \alpha^{2}}{16 \omega^{2}} J_{10}+\frac{\gamma \lambda \alpha^{2}}{\omega^{2}} J_{8}+\frac{\lambda^{2} \alpha^{2}}{\omega^{2}} J_{6}\right)
$$

where

$$
\alpha=\frac{2 h\left(\omega^{2}+\varepsilon \lambda\right)}{\sigma^{2}}
$$

The expressions $J_{i}(i=0,2,4, \ldots)$ are shown in the Appendix 1 .

Substituting $J_{6}, J_{8}, J_{10}$ and (3.6) into (3.5) one gets, finally

$$
\begin{aligned}
H(\alpha)= & \pi \sqrt{2 \pi} c^{2}\left\{\frac{4725 \gamma^{2}}{2^{16} \omega^{2}} \frac{1}{\alpha^{3} \sqrt{\alpha}}-\frac{105}{2^{10} \varepsilon} \frac{1}{\alpha^{2} \sqrt{\alpha}}\right. \\
& \left.+\left(\frac{105}{2^{11} \omega^{2} \varepsilon} \frac{\sigma^{2}}{h}+\frac{15 \omega^{2}}{2^{8} \varepsilon^{2}}\right) \frac{1}{\alpha \sqrt{\alpha}}-\frac{15}{2^{8} \varepsilon}\left(\frac{\sigma^{2}}{h}\right)^{2} \frac{1}{\sqrt{\alpha}}+\frac{15}{2^{10} \omega^{2} \varepsilon^{2}}\left(\frac{\sigma^{2}}{h}\right)^{2} \sqrt{\alpha}\right\}
\end{aligned}
$$


In order to determine the smallest value of the function $H(\alpha)$ in the interval $\alpha \in(0,+\infty)$ we consider the following equation

$$
\begin{aligned}
\frac{d H(\alpha)}{d \alpha}= & \pi \sqrt{\pi} c^{2}\left\{-\frac{33075 \gamma^{2}}{2^{17} \omega^{2}} \frac{1}{\alpha^{4} \sqrt{\alpha}}+\frac{525}{2^{11} \varepsilon} \frac{1}{\alpha^{3} \sqrt{\alpha}}-\left(\frac{315}{2^{12} \omega^{2} \varepsilon} \frac{\sigma^{2}}{h}\right.\right. \\
& \left.\left.+\frac{45 \omega^{2}}{2^{9} \varepsilon^{2}}\right) \frac{1}{\alpha^{2} \sqrt{\alpha}}+\frac{15}{2^{9} \varepsilon^{2}}\left(\frac{\sigma^{2}}{h}\right)^{2} \frac{1}{\alpha \sqrt{\alpha}}+\frac{15}{2^{11} \omega^{2} \varepsilon^{2}}\left(\frac{\sigma^{2}}{h}\right)^{2} \frac{1}{\sqrt{\alpha}}\right\}=0 .
\end{aligned}
$$

From the equation (3.8) one obtains the solution $\alpha=\alpha_{\min }>0$ at which the function (3.7) has the smallest value $H_{\min }=H\left(\alpha_{\min }\right)$. The value $\alpha_{\min }$ depending one $\omega, \gamma, \sigma^{2}, h, \varepsilon$ can be determined numerically.

The mean square of displacement corresponding to $\alpha_{\min }$ takes the form

$$
\left\langle x^{2}\right\rangle_{T}=\int_{0}^{2 \pi} \int_{0}^{\infty} x^{2} W_{L}\left(a, \alpha_{\min }\right) d a d \varphi=\frac{1}{2 \alpha_{\min }} .
$$

The result obtained by the expression (3.9) $\left\langle x^{2}\right\rangle_{T}$ is compared in the Tab. 1 with the exact solution for values $\omega=1, \gamma=1, \sigma^{2}=4 h$, and different values of $\varepsilon$. In addition, the result obtained by the classical GEL $\left\langle x^{2}\right\rangle_{g}$ is also shown. Obviously, the solution $\left\langle x^{2}\right\rangle_{T}$ is much closer to the exact

\begin{tabular}{|c|c|c|c|c|}
\hline$N$ & $\varepsilon$ & $\left\langle x^{2}\right\rangle_{e}$ & $\left\langle x^{2}\right\rangle_{g}$ & $\left\langle x^{2}\right\rangle_{T}$ \\
\hline 1 & 0.1 & 0.8176 & $0.8054(1.49 \%)$ & $0.8289(1.38 \%)$ \\
\hline 2 & 1.0 & 0.4679 & $0.4343(7.19 \%)$ & $0.4492(3.99 \%)$ \\
\hline 3 & 10.0 & 0.1889 & $0.1667(11.8 \%)$ & $0.1745(7.57 \%)$ \\
\hline 4 & 100.0 & 0.0650 & $0.0561(13.6 \%)$ & $0.0588(9.54 \%)$ \\
\hline
\end{tabular}
solution $\left\langle x^{2}\right\rangle_{c}$ than the solution $\left\langle x^{2}\right\rangle_{g}$

Table 1. Approximate mean squares of displacement for Duffing equation

\subsection{Van der Pol oscillator}

As a second example we consider a Vanderpol oscillator

$$
\ddot{x}+\omega^{2} x+\varepsilon\left(\gamma x^{2}-1\right) \dot{x}=\sigma \dot{\xi}(t) .
$$

In this case one gets

$$
f(x, \dot{x})=\left(\gamma x^{2}-1\right) \dot{x}, \quad \gamma=\text { const },
$$

and the linearized equation takes the form

$$
\ddot{x}+\varepsilon \mu \dot{x}+\omega^{2} x=\sigma \dot{\xi}(t) .
$$

So, one gets the following probability density function

$$
W_{L}(a, \mu)=c a \exp \left\{-\frac{\omega^{2} \varepsilon \mu}{\sigma^{2}} a^{2}\right\} .
$$

Substituting (3.11), (3.13) into (2.12) and noting (2.5), after some calculations one obtains

$$
\begin{aligned}
H(\beta)= & \pi \sqrt{2 \pi} c^{2}\left[\frac{29 \sigma^{4}}{2^{8} \varepsilon^{2} \omega^{4}} \sqrt{\beta}+\frac{29 \sigma^{2}}{2^{7} \varepsilon \omega^{2}} \frac{1}{\sqrt{\beta}}\right. \\
& \left.+\left(\frac{29}{2^{8}}+\frac{249 \gamma \sigma^{2}}{2^{10} \varepsilon \omega^{2}}\right) \frac{1}{\beta \sqrt{\beta}}+\frac{249 \gamma}{2^{10}} \cdot \frac{1}{\beta^{2} \sqrt{\beta}}+\frac{9375 \gamma^{2}}{2^{16}} \cdot \frac{1}{\beta^{2} \sqrt{\beta}}\right]
\end{aligned}
$$


where

$$
\beta=\frac{\omega^{2} \varepsilon \mu}{\sigma^{2}} .
$$

Differentiating the function (3.14) with respect to $\beta$ one gets the following equation

$$
\begin{aligned}
\frac{d H(\beta)}{d \beta}= & \pi \sqrt{\pi} c^{2}\left[\frac{29 \sigma^{4}}{2^{9} \varepsilon^{2} \omega^{4}} \cdot \frac{1}{\sqrt{\beta}}-\frac{29 \sigma^{2}}{2^{8} \varepsilon \omega^{2}} \cdot \frac{1}{\beta \sqrt{\beta}}-\left(\frac{87}{2^{9}}+\frac{747 \gamma \sigma^{2}}{2^{11} \varepsilon \omega^{2}}\right) \frac{1}{\beta^{2} \sqrt{\beta}}\right. \\
& \left.-\frac{1245 \gamma}{2^{11}} \cdot \frac{1}{\beta^{3} \sqrt{\beta}}-\frac{65625 \gamma^{2}}{2^{17}} \cdot \frac{1}{\beta^{4} \sqrt{\beta}}\right]=0 .
\end{aligned}
$$

The equation (3.16) gives the solution $\beta=\beta_{\min }>0$ at which the function (3.14) has the smallest value $H_{\min }=H\left(\beta_{\min }\right)$ in the interval $\beta \in(0,+\infty)$. The result obtained from the expression similar to the one (3.9) $\left\langle x^{2}\right\rangle_{T}$ is compared in the Tab. 2 with the simulation solutions $\left\langle x^{2}\right\rangle_{M C}$ taken in [Roy and Spanos, 1991] where $\omega=1, \gamma=10, \varepsilon=0.2$ and for different values of $\sigma^{2}$. In addition, the resalts obtained by the classical GEL and the classical SAM (Stochastic averaging method) $\left\langle x^{2}\right\rangle_{S A M}$ [Roy \& Spanos, 1991] are also shown. It is seen from the Table 2 that the solution $\left\langle x^{2}\right\rangle_{T}$ is much closer to the simulation $\left\langle x^{2}\right\rangle_{M C}$ than the solution $\left\langle x^{2}\right\rangle_{g}$

Tab. 2. Approximate mean squares of displacement Van der Pol equation

\begin{tabular}{ccccccc}
\hline$N$ & $\sigma^{2}$ & & $\left\langle x^{2}\right\rangle_{M C}$ & $\left\langle x^{2}\right\rangle_{g}$ & $\left\langle x^{2}\right\rangle_{S A M}$ & \\
\cline { 1 - 2 } 1 & 0.02 & 0.2080 & $0.137(34 \%)$ & $0.2055(1.2 \%)$ & $0.1859(10.62 \%)$ \\
2 & 0.2 & 0.3600 & $0.279(22 \%)$ & $0.3402(5.5 \%)$ & $0.3445(4.49 \%)$ \\
3 & 1.0 & 0.7325 & $0.552(25 \%)$ & $0.6433(12.1 \%)$ & $0.6903(9.17 \%)$ \\
4 & 2.0 & 1.0310 & $0.759(26 \%)$ & $0.8750(15.1 \%)$ & $0.9152(11.23 \%)$ \\
5 & 4.0 & 1.4540 & $1.051(28 \%)$ & $1.2040(17.2 \%)$ & $1.2916(11.17 \%)$ \\
\hline
\end{tabular}

\section{Conclusions}

The problem for discovering new techniques to overcome the limitation of the classical GEL for both weak and strong non-linearity is of great interest. In the paper a technique to treat stationary response of non-linear systems under a zero mean Gaussian white noise is presented based on the Fokker - Plank equation approach. The proposed technique is then applied to Duffing and Van der Pol oscillators to show significant improvements over the accuracy of classical GEL.

Acknowledgement. Support from the fundamental research Project.in natural sciences is gratefully acknowledged.

\section{Appendix}

1. Let

$$
J_{2 n}=\int_{0}^{\infty} a^{2 n} e^{-2 \alpha a^{2}} d a ; \quad(\alpha>0, n=0,1,2, \ldots)
$$

one gets

$$
J_{0}=\frac{\sqrt{2 \pi}}{4 \alpha}
$$

and

$$
J_{2 n+2}=\frac{2 n+1}{4 \alpha} J_{2 n} \quad(n=0,1,2, \ldots) .
$$




\section{Property}

Let the function $y=f(x)$ be defined, continuously in the interval $x \in(0,+\infty)$ and satisfy the following conditions

$$
\lim _{x \rightarrow 0} f(x)=+\infty ; \quad \lim _{x \rightarrow+\infty} f(x)=+\infty .
$$

It is known that the function $f(x)$ has the smallest value in the interval $(0,+\infty)$. In particular, the functions $H(\alpha)$ in (19) and $H(\beta)$ in (26) have the property mentioned above, so both of them have the smallest values in the interval $(0,+\infty)$.

\section{References}

1. Mitropolskii Yu. A., Dao N. V., Anh N. D. Non-linear oscillations in systems of arbitrary order, Naukova dumka, Kiev 1992 (in Russian).

2. Roy R. V., Spanos P. D. Pade-type approach to non-linear random vibration analysis, Probabilistic Engineering Mechanic, part 2, 6, No 3, 1991, 119-128.

3. Socha L., Soong T. T. Linearization in analysis of non-linear stochastic systems, ASME J. Appl. Math. Rev., 44 No 10, 1991, 399-422.

4. Caughey T. K. Response of a non-linear string to random loading, J. App., 26, 1959, 341-344.

5. Anh N. D., Di Paola M. Some extensions of Gaussian equivalent linearization, Proc. Int. Conf. On Non-linear Stochastic Dynamics in Hanoi, Vietnam, December, 1995.

6. Anh N. D., Cuong D. A., Thinh N. H. On the probabilistic criterion for Gaussian equivalent linearization. Proc. Int. Conf. On Non-linear Stochastic Dynamics in Hanoi, Vietnam, December, 1995.

7. Anh N. D., W. Schiehlen W. Probabilistic criterion for Gaussian equivalent linearization. Journal of Mechanics, T.18, No 2, 1996, 1-6.

8. Socha L. Application of probability metrics to the linearization and sensivity analytic of stochastic dynamic systems, Proc. Int. Conf. On Non-linear Stochastic Dynamics in Hanoi, Vietnam, December 1995.

Received December 24, 1996

\section{TUYẾN TÍNH HÓA NGẪU NHIÊN TƯƠNG ĐƯONG DƯ A TRÊN PHƯONG TRİNH FOKKER-PLANK}

Để khăc phục những hạn chế cưa phương pháp tuyến tính hóa tương đương Gauss kinh điển trong trường hợp phi tuyến mạnh, các tác giả đã đề nghị một tiêu chuẩn tuyến tính hóa tương đương khác dựa trên việc khảo sát phương trình Fokker-Plank đối với hàm mật độ xác suất dừng của biên độ và pha. Kết quả áp dụng cho các hệ Duffing và Van der Pol cho kết quá tốt hơn đối với phương pháp tuyến tính hóa kinh điển. 